\title{
Global Phosphoproteomic Analysis Reveals Significant Metabolic Reprogramming in the Termination of Liver Regeneration in Mice
}

\author{
Jingzi Zhang ${ }^{1, \ddagger}$, Neng Tang ${ }^{4, \ddagger}$, Yinjuan Zhao ${ }^{3}$, Ruoyu Zhao ${ }^{1}$, Xiao Fu${ }^{4}$, Dandan Zhao ${ }^{1}$, Yue \\ Zhao $^{1}$, Lan Huang ${ }^{5}$, Chaojun $\mathrm{Li}^{1}$, Yudong Qiu ${ }^{4,},{ }^{*}$, Bin Xue ${ }^{2,{ }^{*}}$, Lei Fang ${ }^{1,{ }^{*}}$ \\ ${ }^{1}$ Model Animal Research Center and Medical School of Nanjing University, Nanjing 210093, \\ China \\ ${ }^{2}$ Core Laboratory, Sir Run Run Hospital, Nanjing Medical University, Nanjing, 211166, China \\ ${ }^{3}$ Collaborative Innovation Center of Sustainable Forestry in Southern China, College of Forestry, \\ Nanjing Forestry University, Nanjing 210037, China \\ ${ }^{4}$ Nanjing Drum Tower Hospital, Medical School of Nanjing University, Nanjing 210093, China \\ ${ }^{5}$ Department of Physiology and Biophysics, University of California, Irvine, CA 92697, USA
}

\section{Abstract}

\begin{abstract}
Phosphorylation is crucial in regulating various biological processes. However, comprehensive phosphoproteomic profiling in the termination of liver regeneration (LR) is still missing. Here, we used TMT labeling coupled with phosphopeptides enrichment and 2D LC-MS/MS analysis to establish a global phosphoproteomic map in the liver of mice at day 5 after partial hepatectomy (PH). Altogether, 9731 phosphosites from 3443 proteins were identified and 7802 phosphosites from 2980 proteins were quantified. Motif analysis of the identified phosphosites revealed a diverse array of consensus sequences, suggesting multiple kinase families including ERK/MAPK, PKA/PKC, CaMK-II, CKII and CDK may be involved in the termination of LR. Functional clustering analysis of proteins with dysregulated phosphosites showed that they mainly participate in metabolic pathways, DNA replication and tight junction. More importantly, deletion of PP2Aca in the liver remarkably changes the overall phosphorylation profile, indicating its critical role in regulating the termination of LR. Finally, several differentially phosphorylated sites were validated by Co-immunoprecipitation and Western blot. Taken together, our data unravels the first comprehensive phosphoproteomic map in the termination of LR in mice, which greatly expands
\end{abstract}

\footnotetext{
*Correspondence should be addressed to: Dr. Yundong Qiu (yudongqiu510@163.com), 321 Zhongshan Road, Gulou District, Nanjing, 210008, People's Republic of China. Phone: +86-25-83596845, Fax: +86-25-83596845.; Dr. Bin Xue (xuebin@ @ju.edu.cn), 22 Hankou Road, Gulou District, Nanjing University, Nanjing, 210093, People's Republic of China. Phone: +86-25-83596845, Fax: +86-25-83596845.; Dr. Lei Fang (njfanglei@ nju.edu.cn), 22 Hankou Road, Gulou District, Nanjing University, Nanjing, 210093, People's Republic of China. Phone: +86-25-83596845, Fax: +86-25-83596845.

*These authors contributed equally.

Author Contributions

The manuscript was written through contributions of all authors. All authors have given approval to the final version of the manuscript.

Supporting Information

The following supporting information is available free of charge at ACS website http://pubs.acs.org.
} 
our knowledge in the complicate regulation of this process and provides new directions for the treatment of liver cancer using liver resection.

\section{Graphical Abstract}
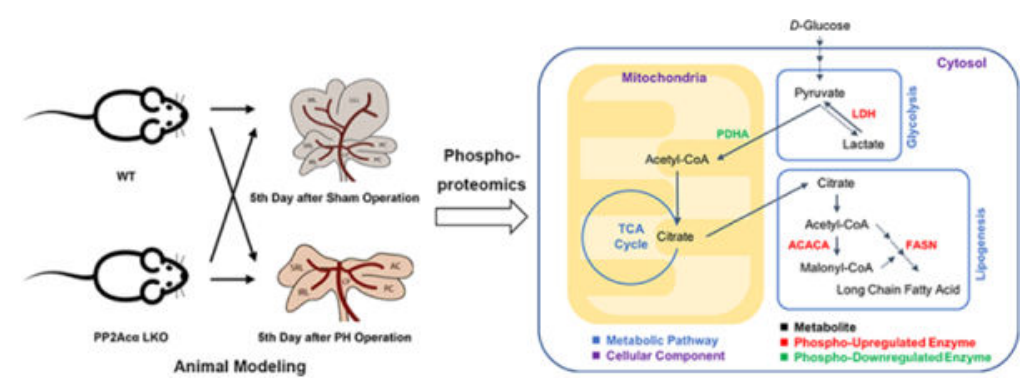

\section{Keywords}

Phosphorylation; partial hepatectomy; liver regeneration; TMT; PP2Aca; metabolic reprogramming

\section{Introduction}

The liver is the only organ that has the ability to regenerate after massive loss of tissue mass induced by partial hepatectomy $(\mathrm{PH})$ or toxic injury ${ }^{1}$. Liver resection is commonly performed for primary and metastatic liver cancer and is considered as the most effective method to treat liver cancer ${ }^{2,3}$. Understanding the hepatic regenerative process is clinically important as effective treatments of chronic liver diseases, such as resection of tumors and donor liver transplantation, requires complete liver regeneration. This process is marked by how well the architecture, size and function of the liver can be restored. Hepatocytes undergo replication to restore the liver mass by compensatory hyperplasia. Immediately after hepatectomy, the liver begins to regenerate ${ }^{1}$. Cytokines, growth factors, and metabolic networks are essential components required for liver regeneration. These include changes in the enzymatic activation of extracellular matrix remodelers (e.g. urokinase plasminogen activator, MMP-9 $)^{4}$, relative changes in blood flow volume in relation to the remaining hepatic sinusoid, the increment on the bioavailability of certain growth factors (HGF, EGFR ligands) ${ }^{5}$, hormones (noradrenalin, serotonin) and cytokines ${ }^{6}$; the activation of transcription factors (STAT3, NF- $\kappa B$, Notch-NICD, $\beta$-catenin) and changes in the transcriptome of hepatocytes ${ }^{7,8}$.

Post-translational modifications (PTMs) provide a means to regulate protein function, and phosphorylation is one of ubiquitous and extensively studied PTMs ${ }^{9,10}$. Reversible protein phosphorylation temporally and spatially modifies $30 \%$ of all cellular proteins and plays a crucial role in regulating a variety of biological processes such as signal transduction and the cell cycle, and controls various biological functions like cell proliferation, growth, polarization, survival and apoptosis ${ }^{10}$. The presence of multiple phosphosites within a protein expands the possibilities and complexities for regulation. Phosphorylation at different sites within a protein may have similar or opposite effects on one specific function 
and/or distinct functions of the protein ${ }^{11-13}$. Phosphate groups can be directly involved in ligand binding. Phosphorylation/dephosphorylation can also be considered to be an on/off switch for ligand interaction and protein function when the phospho-group is directly involved in molecular interactions ${ }^{10,14}$. Thus, the identification of kinase-specific phosphosites and the systematic elucidation of site-specific kinase-substrate relations would provide a fundamental basis for understanding cellular pathways to unravel molecular mechanisms underlying human pathology, which would help identifying potential drug targets for future biomedical design ${ }^{15,16}$.

During the liver regeneration process, many key signaling pathways are regulated by phosphorylation, such as STAT3, YAP, MAPK, Akt, GSK-3 $\beta$, AMPK and NF- $\mathrm{kB}^{17-21}$. After partial hepatectomy, hepatocytes proliferate rapidly, correlating with increased AMPK phosphorylation. Apart from negative regulation of $\beta$-catenin, GSK3 can directly regulate several other cell proliferation mediators, including cyclin D1, c-Myc, c-Jun, and eIF2B ${ }^{20}$. Thus, GSK3 could also potentially alter liver regeneration. Upon binding of TGF- $\beta$ to its type I and II surface receptor complexes, intracellular Smad2 and 3 are phosphorylated and form a complex with Smad4, which translocate to the nucleus to modulate the transcriptional response by interacting with a variety of factors regulating the expression of target genes ${ }^{22}$. Stat 3 signaling is also a known regulator of cell survival and proliferation. Upon activation by a number of factors including IL-6, Stat3 promotes cell survival including the induction of anti-apoptotic factors while also promoting cell cycle progression and liver regeneration ${ }^{8}$.

Up to now, mechanisms leading to termination of liver regeneration remain elusive. TGF- $\beta$ is usually considered as the termination signal of liver regeneration ${ }^{6,23}$. Satellite cells which are activated by TGF- $\beta$ secret large amount of extracellular matrix. Newly synthesized matrix binds HGF and prevents it from being activated by urokinase. Besides TGF- $\beta$, normalized blood flow of the liver may contribute to the termination of regeneration. Protein phosphatases 1 and 2A (PP1 and PP2A) are the most ubiquitous and abundant serine/ threonine phosphatases in eukaryotic cells ${ }^{24}$. PP2A plays a critical role in the regulation of the cell cycle. It is known to dephosphorylate over 300 substrates involved in the cell cycle, regulating almost all major pathways and cell cycle checkpoints. We have previously reported that PP2Aca-/- mice showed prolonged LR termination and an increased liver size compared to the original mass ${ }^{25}$. Further study demonstrated PP2Aca plays an essential role in the proper termination of LR via the AKT/GSK3 $\beta /$ Cyclin D1 pathway, indicating that phosphorylation/dephosphorylation events play critical roles in controlling termination of liver regeneration. However, comprehensive investigation of global protein phosphorylation/ dephosphorylation and the role of PP2Aca in this process are still missing. In this study, we report an integrated approach of TMT labeling coupled with phosphopeptides enrichment and 2D LC-MS/MS to establish a global phosphoproteomic profile in the termination of LR in both wild type and PP2Aca-/- mice using $70 \%$ partial hepatectomy model. 


\section{Material and methods}

\section{Mice PH model and liver tissue preparation}

This study was approved by the Ethics Committee of the Affiliated Drum Tower Hospital of Medical School, Nanjing University. C57BL6/J mice were bought from Model Animal Research Center of Nanjing University. PP2Aca-/- mice were generated as described previously $^{25}$. Adult male C57BL6/J (Wild-type) and PP2Aca-/- mice which were 8-10 weeks old were used in our study. The mice were housed in a temperature-controlled animal facility with 12-h light-dark cycles and had unlimited access to water and commercial food. To perform partial hepatectomy, animals were anesthetized by inhalation of isoflurane. The abdomen was accessed via midline laparotomy, the left lateral and median lobes (70 \% of the liver) were ligated separately with a single $4-0$ silk suture and resected. The abdomen was closed with also a 4-0 silk suture and mice were placed in an incubator $\left(37^{\circ} \mathrm{C}\right)$ for recovery. Sham operations comprised the same procedure but omitted removal of the liver lobes. The mice were sacrificed at 5 days after hepatectomy ( $\mathrm{n}=3$ at each time point) which represents the key time point of liver regeneration termination by our previous study ${ }^{25}$. The liver/body weight ratio was measured and liver tissues were snap frozen in liquid nitrogen and stored in $-80{ }^{\circ} \mathrm{C}$ for further analysis.

\section{Protein extraction}

Mice liver tissue was grinded by liquid nitrogen into cell powder and then transferred to a 5$\mathrm{mL}$ centrifuge tube. After that, four volumes of lysis buffer $(8 \mathrm{M}$ urea, $1 \%$ Protease Inhibitor Cocktail) were added to the cell powder, followed by sonication three times on ice using a high intensity ultrasonic processor (Scientz). The remaining debris was removed by centrifugation at $12,000 \mathrm{~g}$ at $4{ }^{\circ} \mathrm{C}$ for $10 \mathrm{~min}$. Finally, the supernatant was collected and the protein concentration was determined with Pierce ${ }^{\mathrm{TM}} \mathrm{BCA}$ Protein Assay Kit (Thermo Scientific) according to the manufacturer's instructions.

\section{Trypsin digestion and TMT Labeling}

For digestion, $1 \mathrm{mg}$ protein solution was reduced with $5 \mathrm{mM}$ dithiothreitol for $30 \mathrm{~min}$ at 56 ${ }^{\circ} \mathrm{C}$ and alkylated with $10 \mathrm{mM}$ iodoacetamide for $15 \mathrm{~min}$ at room temperature in darkness. The protein sample was then diluted by adding $100 \mathrm{mM}$ TEAB to make urea concentration less than 2M. Finally, trypsin was added at 1:50 trypsin-to-protein mass ratio for the first digestion overnight and 1:100 trypsin-to-protein mass ratio for a second $4 \mathrm{~h}$-digestion. After trypsin digestion, peptide was desalted by Strata X C18 SPE column (Phenomenex) and vacuum-dried. Peptide was reconstituted in $0.5 \mathrm{M}$ TEAB and processed according to the manufacturer's protocol for TMT 6-plex kit (Thermo). Briefly, one unit of TMT reagent were thawed, reconstituted in acetonitrile and added to the peptides. The peptide mixtures were then incubated for $2 \mathrm{~h}$ at room temperature and pooled, desalted and dried by vacuum centrifugation.

\section{HPLC fractionation}

The tryptic peptides were fractionated into fractions by high $\mathrm{pH}$ reverse-phase HPLC (Agilent 1260) using Thermo Betasil C18 column (5 $\mu \mathrm{m}$ particles, $10 \mathrm{~mm}$ ID, $250 \mathrm{~mm}$ 
length). The buffers used for basic reverse phase were buffer A ( $2 \%$ acetonitrile, $10 \mathrm{mM}$ ammonium formate, $\mathrm{pH} 9$ ) and buffer $\mathrm{B}$ (98\% acetonitrile, 10mM ammonium formate, $\mathrm{pH}$ 9). Briefly, peptides were first separated with a gradient of $8 \%$ to $32 \%$ acetonitrile ( $\mathrm{pH} 9$ ) over $40 \mathrm{~min}$ into 40 fractions at a flow rate of $1 \mathrm{~mL} / \mathrm{min}$. Then, the peptides were combined into 8 samples by pooling fractions with every 8 intervals, e.g. pooling fractions $(1,9,17$, $25,33)$ as sample 1 , fractions $(2,10,18,26,34)$ as sample 2 , until fractions $(8,16,24,32$, 40 ) as sample 8 . The combined samples were then dried by vacuum centrifugation for next step.

\section{Affinity enrichment of phosphopeptides}

IMAC microspheres were bought from Dalian Institute of Chemical Physics, Chinese Academy of Sciences and $\mathrm{Ti}^{4+}{ }^{4}$-IMAC microsphere was used here to enrich phosphopeptide ${ }^{26}$. Peptide mixtures were first incubated with IMAC microspheres suspension with vibration in loading buffer (50\% acetonitrile/6\% trifluoroacetic acid). The IMAC microspheres with enriched phosphopeptides were collected by centrifugation, and the supernatant was removed. To remove nonspecifically adsorbed peptides, the IMAC microspheres were washed with 50\% acetonitrile/6\% trifluoroacetic acid and 30\% acetonitrile/ $0.1 \%$ trifluoroacetic acid, sequentially. To elute the enriched phosphopeptides from the IMAC microspheres, elution buffer containing $10 \% \mathrm{NH}_{4} \mathrm{OH}$ was added and the enriched phosphopeptides were eluted with vibration. The supernatant containing phosphopeptides was collected and lyophilized for LC-MS/MS analysis.

\section{LC-MS/MS analysis}

The tryptic peptides of each fraction were dissolved in solvent A ( $0.1 \%$ formic acid in $2 \%$ acetonitrile), directly loaded onto a home-made reversed-phase analytical column $(15 \mathrm{~cm}$ length, $75 \mu \mathrm{m}$ i.d.) packed with Reprosil-Pur Basic C18, $1.9 \mu \mathrm{m}$ (Dr. Maisch GmbH HPLC). The gradient was comprised of an increase from $2 \%$ to $22 \%$ solvent B $(0.1 \%$ formic acid in 98\% acetonitrile) over $40 \mathrm{~min}, 22 \%$ to $35 \%$ in $12 \mathrm{~min}$ and climbing to $80 \%$ in $4 \mathrm{~min}$ then holding at $80 \%$ for the last $4 \mathrm{~min}$, all at a constant flow rate of $350 \mathrm{~nL} / \mathrm{min}$ on an EASY-nLC 1000 UPLC system.

The peptides were subject to NSI source followed by tandem mass spectrometry (MS/MS) in Q Exactive ${ }^{\mathrm{TM}}$ Plus (Thermo) coupled online to the UPLC. The electrospray voltage applied was $2.0 \mathrm{kV}$. The $\mathrm{m} / \mathrm{z}$ scan range was 350 to 1800 for full scan, and intact peptides were detected in the Orbitrap at a resolution of 70,000. Peptides were then selected for MS/MS using NCE setting as 28 and the fragments were detected in the Orbitrap at a resolution of 17,500. A data-dependent procedure that alternated between one MS scan followed by $20 \mathrm{MS} / \mathrm{MS}$ scans with $15.0 \mathrm{~s}$ dynamic exclusion. Automatic gain control (AGC) was set at $5 \mathrm{E}^{4}$. Fixed first mass was set as $100 \mathrm{~m} / \mathrm{z}$.

\section{Database search}

The mass spectrometry proteomics data have been deposited to the ProteomeXchange Consortium via the PRIDE ${ }^{27}$ partner repository with the dataset identifier PXD011374. Submission details: Project Name: Global Phosphoproteomic Analysis Reveals Significant Metabolic Reprogramming in the Termination of Liver Regeneration in Mice. Project 
accession: PXD011374; Project DOI: Not applicable. Reviewer account details: Username: reviewer31869@ebi.ac.uk; Password: bHVUE0WF. The resulting MS/MS data were processed using Maxquant search engine (v.1.5.2.8). Tandem mass spectra were searched against UniProt_Mus musculus (83,099 sequences, released on Dec. 2017) database concatenated with reverse decoy database. TMT-4plex was selected as quantification method. Trypsin/P was specified as cleavage enzyme allowing up to 3 missing cleavages, 4 modifications per peptide and 5 charges. Mass error was set to $20 \mathrm{ppm}$ for first search, 5 ppm for main search and 0.02 Da for fragment ions. Carbamidomethylation on Cys was specified as fixed modification and oxidation on Met, phosphorylation on Ser, Thr, Tyr and acetylation on protein $\mathrm{N}$-terminal were specified as variable modifications. False discovery rate (FDR) thresholds for protein, peptide and modification site were specified at $1 \%$. Minimum peptide length was set at 7. All the other parameters in MaxQuant were set to default values. The site localization probability was set as $>0.75$.

\section{Statistical analysis}

For TMT quantification of a specific phosphosite, the ratios of the TMT reporter ion intensities in MS/MS spectra $(\mathrm{m} / \mathrm{z} 126,128,130,131)$ from raw data sets were used to calculate fold changes among samples. Differential expression of phosphosites was calculated by the mean of the three repeats over the mean of the three repeats. For statistical test, two-sample, two-sided T-tests were used to compare the dysregulated phosphosites. In general, $p<0.05$ was considered to be statistically significant, and we reported the $p$ value or significance level any time a statistical test was performed.

\section{Motif analysis}

Software motif- $\mathrm{X}^{28}$ was used to analyze the model of sequences constituted with amino acids in specific positions of modify-13-mers (6 amino acids upstream and downstream of the site) in all protein sequences. And all the database protein sequences were used as background database parameter, other parameters with default.

\section{Enrichment analysis of Gene Ontology, KEGG pathway and protein domain}

Enrichment analysis of Gene Ontology, the Kyoto Encyclopedia of Genes and Genomes (KEGG) pathway and protein domain was performed for proteins with dysregulated phosphosites as described ${ }^{29}$. Briefly, proteins with dysregulated phosphosites were classified by GO annotation into three categories: biological process, cellular compartment and molecular function. Similarly, the KEGG database was used to identify enriched pathways and InterPro database was researched to identify enriched protein domains in proteins with dysregulated phosphosites against all identified proteins. For each GO, KEGG pathway or protein domain, a two-tailed Fisher's exact test was employed to test the enrichment of proteins with dysregulated phosphosites against all identified proteins. The GO, KEGG pathway or protein domain with a corrected $p$ value $<0.05$ is considered significant.

\section{Enrichment-based clustering}

For further hierarchical clustering based on different protein functional classification (such as: Motif, GO, Domain and KEGG Pathway). We first collated all the categories obtained 
after enrichment along with their $p$ values, and then filtered for those categories which were at least enriched in one of the clusters with $p$ value $<0.05$. This filtered $p$ value matrix was transformed by the function $\mathrm{x}=-\log 10$ ( $p$ value). Finally, these $\mathrm{x}$ values were $\mathrm{z}$-transformed for each functional category. These $\mathrm{z}$ scores were then clustered by one-way hierarchical clustering (Euclidean distance, average linkage clustering) in Genesis. Cluster membership was visualized by a heatmap using the "heatmap.2" function from the "gplots" R-package.

\section{Validation of proteins with dysregulated phosphosites in the termination of liver regeneration}

Several proteins were selected for validation from proteins with dysregulated phosphosites identified by TMT quantitation. For validation of PDHA, total lysates of mice liver from different days after PH were used to detect change of PDHA phosphorylation using p-PDHA Ser232 antibody. For validations of other proteins, Co-Immunoprecipitation (Co-IP) was performed according to the manufacturer's instructions (Pierce, USA). Briefly, every $50 \mathrm{mg}$ liver tissue was homogenized in $1 \mathrm{~mL}$ lysis buffer with protease inhibitors and phosphatase inhibitor (Roche, Switzerland). After centrifugation at 12,000g/min for $20 \mathrm{~min}$, the supernatants were harvested as total lysates. After incubating with the Control Agarose Resins to pre-clear, the total lysates were ready to use. $10 \mu \mathrm{g}$ antibodies of ACACA (BM4414, Boster), FASN (PB0909, Boster), GBE1 (20313-1-AP, Proteintech), MAP4K4 (55247-1-AP, Proteintech), Stathmin (11157-1-AP, Proteintech) and BNIP3 (BM5039, Proteintech) were immobilized to the AminoLink Plus Coupling Resins in the columns, respectively. Two columns of each antibody were prepared. Co-IP was performed by adding total lysates from Sham and $\mathrm{PH}$ group respectively into the resins conjugated with different antibodies. After incubating overnight at $4{ }^{\circ} \mathrm{C}$ with gentle mixing, the proteins were eluted from the resins and analyzed by Western blot. The eluted proteins were separated by $10 \%$ SDS-polyacrylamide gel and transferred onto polyvinylidene fluoride membrane. The membrane was probed with Phospho-(Ser/Thr) Phe (Abcam, USA) antibody to detect phosphorylated ACACA, FASN, GBE1, MAP4K4, Stathmin and BNIP3 using an enhanced chemiluminescence Western blotting Kit (Beyotime Biotech, Shanghai, China). The amount of PDHA, ACACA, FASN, GBE1, MAP4K4, Stathmin and BNIP3 in total lysates was also measured by Western blot using corresponding antibody, and $\beta$-Actin (BM0627, Boster) was used as loading control. All the Western blots have been repeated at least three times in our study.

\section{Results}

\section{A schematic workflow of global phosphoproteomic analysis in the termination of mice liver regeneration}

A schematic workflow in this study has been illustrated (Fig. 1). Briefly, 70\% partial hepatectomy was performed for wild-type and PP2Aca-/- mice, named PH-WT and PHKO, respectively. Meanwhile, Sham operation was also performed, and the samples were named Sham-WT and Sham-KO, respectively. Three biological replicates were set, and each replicate contains a group of Sham-WT, PH-WT, Sham-KO and PH-KO. Five days after PH or Sham operation, the livers from all mice were collected and preceded to protein extraction separately. After trypsin digestion, the resulting peptides from each group of Sham-WT, PH- 
WT, Sham-KO and PH-KO were labeled with reagents 126, 128, 130 and 131 of TMT 6plex kit (Thermo, USA), respectively and then mixed prior to high pH HPLC fractionation. Twelve HPLC samples were pooled for IMAC phosphopeptide enrichment and subsequent LC-MS/MS analysis. Then, phosphorylation sites were identified and quantified through database searching, and further subject to extensive bioinformatics analyses to reveal the markedly phosphoproteomic alterations in the termination of liver regeneration in mice.

\section{Quantification overview of phosphorylation sites}

To ensure the validity of MS data, three biological replicates were used in this study. The results have shown high reproducibility among three biological replicates with a Pearson correlation coefficient $>0.8$ (Fig. S-1A). Secondly, the mass error of most peptides is $<5$ ppm, which means the mass accuracy of the MS data fit the requirement (Fig. S-1B). Finally, for most of identified proteins, their number of modification sites per protein was 1 to 3 (Fig. S-1C).

In total, 9,731 phosphosites from 3,443 proteins were identified, among which 7,802 phosphosites from 2,980 proteins were quantified (Fig. 2A, Table S-1). When setting the ratio $>1.2, p<0.05$ as up-regulation and ratio $<1 / 1.2(0.833), p<0.05$ as down-regulation, 754 phosphosites from 506 proteins were up-regulated and 600 phosphosites from 417 proteins were down-regulated in PH-WT/Sham-WT(Fig. 2B and Table S-2), suggesting the remarkably global phosphorylation changes in the termination of mice liver regeneration. Interestingly, 539 phosphosites from 388 proteins were up-regulated and 420 phosphosites from 327 proteins were down-regulated in $\mathrm{PH}-\mathrm{KO} / \mathrm{PH}-\mathrm{WT}$, indicating that the loss of PP2A leaded to markedly phosphoproteomic alterations in the termination of mice liver regeneration.

\section{Motif analysis of all identified phosphosites}

Phosphorylation motifs among all identified phosphosites were analyzed by Motif-x online tool. Totally 22 different motifs were found with fold increase $>10$, including 12 Ser and 10 Thr motifs (Fig. 3A). These phosphorylation motifs contain common consensus phosphorylation sequences of ERK/MAPK, PKA/PKC, CaMK-II, CKII and CDK, suggesting that several specific kinase families are involved in the termination of liver regeneration (Fig. 3B and 3C). Among these protein kinases, almost all of them have been reported to participate in cell proliferation, cell cycle, cell skeleton and cell-cell adhesion, suggesting these kinases might play essential roles in regulating the termination of liver regeneration.

\section{Protein annotation of identified and quantified phosphosites}

To further understand the functions and characteristics of proteins with identified and quantified phosphosites, we performed several bioinformatics analyses to annotate their Gene Ontology, Protein Domain, KEGG Pathway and Subcellular Localization. Fig. S-2 displays such annotations for proteins with up-regulated or down-regulated phosphosites, respectively. 
In the proteins with up-regulated phosphosites, regulation of cellular process, regulation of biological process, macromolecule metabolic process, regulation of metabolic process and multicellular organism development are prominently represented in the category of biological process, accounting for 7\%, 5\%, 4\%, 4\% and 4\%, respectively (Fig. S-2A). In the cellular components category, the top 3 classes are intracellular (23\%), cell periphery (9\%) and organelle lumen (8\%) (Fig. S-2B). In the molecular function category, a great number of proteins are involved in nucleic acid binding (8\%), anion binding (8\%), nucleoside phosphate binding (8\%), cation binding (8\%) and enzyme binding $(6 \%)$, indicating the essential regulatory role of phosphorylation in molecular binding and catalytic activity of enzymes (Fig. S-2C). In the subcellular localization category, the top 3 classes are nucleus (48\%), cytoplasm (21\%) and plasma membrane (11\%), which indicates these up-regulated phosphorylation events mainly take place in nucleus and cytoplasm (Fig. S-2D). It's also notable that $7 \%$ of proteins with up-regulated phosphosites are located in mitochondria, suggesting mitochondria may play important role in regulating the termination of liver regeneration.

Functional classifications of the proteins with down-regulated phosphosites are very similar to those with up-regulated phosphosites. In the category of biological process, regulation of cellular process, macromolecule metabolic process, regulation of metabolic process, regulation of biological process and cellular nitrogen compound metabolic process are also prominently represented, accounting for $7 \%, 5 \%, 5 \%, 4 \%$ and $4 \%$ of all proteins with downregulated phosphosites, respectively (Fig. S-2E). In the cellular components category (Fig. $\mathrm{S}-2 \mathrm{~F})$, the top 3 classes are intracellular (24\%), vesicle (9\%) and organelle lumen (8\%). In the molecular function category (Fig. S-2G), most proteins are involved in binding activity including cation binding (9\%), nucleic acid binding (9\%), anion binding (8\%), nucleoside phosphate binding (7\%), and enzyme binding (7\%). In the subcellular localization category (Fig. S-2H), the top 3 classes are nucleus (42\%), cytoplasm (26\%) and plasma membrane (12\%), mitochondria is the fourth large class which accounts for $8 \%$ of proteins with downregulated phosphosites. The proteins with down-regulated phosphosites are also involved in biological processes of transport (4\%), organic cyclic compound metabolic process (3\%), organic substrate biosynthetic process (3\%) and multicellular organism development (3\%) etc.

\section{Functional enrichment of proteins with differentially regulated phosphosites}

GO-based enrichment analysis was further performed to elucidate the preferred biological functions of proteins with differentially regulated phosphosites in PH-WT vs. Sham-WT (Fig. 4). In proteins with up-regulated phosphosites, the enrichment results of cellular component indicate that phosphorylation in the termination of liver regeneration may prefer to regulate cell-cell junction, anchoring junction, cell junction, adherens junction, and cellcell adherens junction (Fig. 4A). In agreement with the observation above, cell adhesion molecule binding was the most enriched category, followed by protein binding involved in cell-cell adhesion and cadherin binding in category of molecular function. Furthermore, the evaluation of biological process shows the top 5 processes are cell adhesion, biological adhesion, cell-cell adhesion, cytoskeleton organization and cell migration, followed by locomotion. 
In contrary to proteins with up-regulated phosphosites, the enrichment results of proteins with down-regulated phosphosites show distinct characteristics in all 3 GO-based analyses (Fig. 4B). In category of cellular component, endoplasmic reticulum membrane, ribosome, cell cortex part and Golgi membrane are prominently enriched. In category of molecular function, iron ion binding was the most enriched category, followed by oxidoreductase activity and substrate-specific transporter activity. In category of biological process, the top 3 processes are metabolic processes including organonitrogen compound, cellular amide and cofactor, followed by organonitrogen compound biosynthetic process and oxidationreduction process.

To investigate the metabolic processes and pathways in which the differentially phosphorylated proteins are involved in the termination of liver regeneration, KEGG pathway enrichment analysis was performed for the protein with either up- or downregulated phosphosites, respectively. The proteins with up-regulated phosphosites are mapped to pathways with significant $p$ values $(p<0.05)$ including leukocyte transendothelial migration, focal adhesion, tight junction, axon guidance, DNA replication, mismatch repair and regulation of actin cytoskeleton (Fig. 4C). While in proteins with downregulated phosphosites, most of them are mapped to malaria, calcium signaling pathway, glucagon signaling pathway, histidine metabolism and salivary secretion (Fig. 4D).

Protein functions are largely dependent on specific domain structures in the sequence. Thus, we performed domain enrichment analysis to assess the domain structures of proteins with differentially regulated phosphosites in the termination of liver regeneration. The protein domains significantly enriched in the proteins with up-regulated phosphosites includes ubiquitin-related domain, calponin homology domain, guanlylate kinase/L-type calcium channel beta subunit, SH3 domain and PDZ domain, etc. (Fig. 4E). While in proteins with down-regulated phosphosites, the significantly enriched protein domains are P-type ATPase, transmembrane and GRIP domain (Fig. 4F).

\section{Validation of proteins with differentially regulated phosphosites and their biological significance}

To verify the dynamic phosphorylation changes induced by PH in liver regeneration, several proteins with differentially regulated phosphosites including PDHA, ACACA, FASN, GBE1, MAP4K4, Stathmin and BNIP3 were chosen to validate the phosphoproteomic results (Table S-3). Among them, PDHA, ACACA, FASN and GBE1 are important metabolic enzymes; MAP4K4 is a key kinase in MAPK signaling pathway; Stathmin plays crucial roles in preventing assembly and promoting disassembly of microtubules, which is very important in controlling mitotic spindle organization and mitotic cytokinesis; BNIP3 is an apoptosis-inducing protein that can overcome BCL2 suppression, and also involved in mitochondrial quality control.

In our study, in the first 4 days after PH, PDHA was highly phosphorylated at S-232, S-293, S-295 and S-300, possibly by PDK family. Interestingly, at day 5 after PH, the phosphorylation of PDHA rapidly decreased (Table S-3, Fig. 5B). In contrary to PDHA, multiple phosphosites of acetyl-coenzyme carboxylase (ACACA) were up-regulated at day 5 after PH (Table S-3, Fig. 5C), inducing the inhibition of its activity. Similarly, Ser-2403 and 
Thr-976 of FASN were up-regulated 2.11 folds and 1.34 folds in PH-WT/Sham-WT, respectively (Table S-3 and Fig. 5D). We also validated the altered phosphorylation of several other proteins (Fig. 5 E-H) such as 1,4-alpha-glucan-branching enzyme (GBE1), mitogen-activated protein kinase kinase kinase kinase 4 (MAP4K4), Stathmin, and BCL2/ adenovirus E1B $19 \mathrm{kDa}$ protein-interacting protein 3 (BNIP3). These proteins are involved in various biological processes including glycogen accumulation, MAPK signaling pathway, microtubule filament assembly/disassembly and mitochondrial quality control, which are closely related to the termination of liver regeneration.

\section{Dramatic global phosphorylation changes in PP2Aca-/- mice in the termination of liver regeneration}

In our previous work, we've reported that PP2Aca-/- mice showed prolonged LR termination and an increased liver size compared to the original mass via the AKT/GSK3 $\beta$ / Cyclin D1 pathway ${ }^{25}$. In this study, we show that PP2Aca-/- liver specific deletion induces dramatic global phosphorylation changes in the termination of liver regeneration. As shown in Fig. 6A, enrichment-based clustering on KEGG pathway of PH-KO/PH-WT demonstrates distinct pattern compared to PH-WT/Sham-WT. In PH-KO/PH-WT, the proteins with upregulated phosphosites mainly participate in HIF-1 signaling pathway, adherens junction, hypertrophic cardiomyopathy, glucagon signaling pathway, proteoglycans in cancer, regulation of actin cytoskeleton, axon guidance, followed by AMPK signaling pathway and insulin signaling pathway, which are not significantly changed in PH-WT/Sham-WT. Further KEGG pathway analysis of proteins with up-regulated phosphosites in $\mathrm{PH}-\mathrm{KO} / \mathrm{PH}-$ WT shows similar results that proteoglycans in cancer, glucagon signaling pathway, axon guidance, insulin signaling pathway and AMPK signaling pathway are the top 5 enriched pathways (Fig. 6B).

Glucagon signaling pathway plays critical anti-hypoglycemic role in maintaining glucose homeostasis in both animals and humans. To increase blood glucose, glucagon promotes hepatic glucose output by increasing glycogenolysis and gluconeogenesis and by decreasing glycogenesis and glycolysis in a concerted fashion via multiple mechanisms. In WT mice, phosphorylation of key molecules in glucagon signaling pathway including SIK2, IP3R, PHK, PFKFB1, AMPK, LDH and PECK decreases significantly at day 5 after PH compared to Sham group, indicating the inactivation of this pathway in the termination of LR (Fig. 6C). However, this pathway switched to continuous activation by demonstrating remarkably enhanced phosphorylation of these seven proteins in PP2Aca-/- mice compared to WT mice at day 5 after PH, suggesting PP2A is essential for proper termination of LR via regulating glucose metabolism (Fig. $6 \mathrm{C}-\mathrm{D}$ ).

\section{Discussion}

Liver resection is the most common treatment for primary and metastatic liver cancer ${ }^{2,3}$. Immediately after hepatectomy, the liver begins to regenerate. Thus, understanding the hepatic regenerative process has critical clinical interest. Numerous studies have shown liver regeneration is a complex while precisely tuned process, in which cytokines, growth factors, and metabolic networks work synergistically to monitor its initiation, progression and 
termination $^{5,21,22,30}$. As one of the most important post-translational modifications, phosphorylation is well-known in regulating various biological processes such as cell proliferation, growth, polarization, survival and apoptosis ${ }^{13}, 31,32$. Our previous study of liver specific PP2Aca-/- mice has also shown phosphorylation/dephosphorylation events play crucial roles in controlling liver regeneration, especially the termination process. However, comprehensive phosphoproteomic profiling in the termination of liver regeneration is still missing. In this study, we used TMT labeling coupled with phosphopeptides enrichment and 2D LC-MS/MS analysis to establish a global map of protein phosphorylation/dephosphorylation in the termination of liver regeneration in mice. Changes of phosphorylation could be affected by both the protein phosphorylation status and also the protein expression level. Thus, in addition to the phosphoproteomic analysis, future general proteomic analysis in parallel would complement the findings of significantly changed protein phosphorylation in this study.

Based on KEGG pathway analysis, seven proteins with differentially regulated phosphosites such as PDHA, ACACA, FASN, GBE1, MAP4K4, Stathmin and BNIP3, were selected for validating our phosphoproteomic results in PH-WT/Sham-WT. The pyruvate dehydrogenase complex catalyzes the overall conversion of pyruvate to acetyl-CoA and $\mathrm{CO}_{2}$, and thereby links the glycolytic pathway to the tricarboxylic (TCA) cycle. Phosphorylation of pyruvate dehydrogenase (PDHA) abolishes its activity and shuts down the TCA cycle, switching the glucose metabolism from oxidative phosphorylation to glycolysis ${ }^{33}, 34$. Our results show in the first 4 days after PH, PDHA was highly phosphorylated at S-232, S-293, S-295 and S-300 (Table S-3, Fig. 5B) possibly by PDK family, indicating that during the initiation and in the middle of liver regeneration, the glucose metabolism preferred to glycolysis, which mimics the Warburg effects in tumor metabolism. Interestingly, at day 5 after PH, the phosphorylation of PDHA was rapidly decreased (Table S-3, Fig. 5B), and switched glucose metabolism to oxidative phosphorylation in liver. Thus, our data supports that dephosphorylation of PDHA at day 5 after PH might be a key signal in controlling the switch between glycolysis and oxidative phosphorylation to complete the termination of liver regeneration. In contrary to PDHA, multiple phosphosites of acetyl-coenzyme carboxylase (ACACA) were up-regulated at day 5 after PH (Table S-3, Fig. 5C), inducing the inhibition of its activity. ACACA is a biotin-dependent enzyme that catalyzes the irreversible carboxylation of acetyl-CoA to produce malonyl-CoA for the biosynthesis of fatty acids. AMPK is the main kinase regulator of ACACA, able to phosphorylate a number of serine residues on both isoforms of ACACA ${ }^{35}$. However, phosphorylation of AMPK was decreased and AMPK activation was inhibited in our data (Fig. 6A). Further analysis of upregulated phosphosites in ACACA showed consensus sequence of protein kinase CK-II and MAPK, suggesting that some other kinases might be responsible for the phosphorylation of ACACA in the termination of liver regeneration. Fatty acid synthetase (FASN) is a key enzyme in fatty acid biosynthetic process and lipid metabolism by catalyzing the formation of long-chain fatty acids from acetyl-CoA, malonyl-CoA and NADPH. FASN usually forms a homodimer in a head to tail fashion to be active. However, its fatty acid synthase activity is reduced when interacting with CEACAM1 in an insulin and phosphorylation-dependent manner ${ }^{36}$. In our study, the enhanced phosphorylation of FASN in PH-WT/Sham-WT may decrease its fatty acid synthase activity, leading to the inhibition of fatty acid biosynthetic 
process in the termination of liver regeneration (Table S-3, Fig. 5D). Thus, as shown in Fig. 5A, the validation of dysregulated phosphorylation of PDHA, ACACA and FASN, together with the observed increased phosphorylation of L-lactate dehydrogenase A chain (LDH) demonstrates markedly metabolic reprograming including glycolysis, lipogenesis and TCA cycle in regulating the termination of liver regeneration.

We also validated the altered phosphorylation of several other proteins such as mitogenactivated protein kinase kinase kinase kinase 4 (MAP4K4), Stathmin, BCL2/adenovirus E1B $19 \mathrm{kDa}$ protein-interacting protein 3 (BNIP3) and 1,4-alpha-glucan-branching enzyme (GBE1). Importantly, MAP4K4 is a serine/threonine kinase that may play a role in the response to environmental stress and cytokines such as TNF-alpha, induces the MAPK cascade and phosphorylates its downstream substrates like SMAD1. The enhanced phosphorylation of MAP4K4 in our study supports the important role of MAPK signaling pathway in liver regeneration. Taken together, validations of all selected phosphoproteins are highly consistent with our quantitative phosphoproteomic results, demonstrating the successful application of phosphoproteomic approach in mapping the global phosphorylation changes in the termination of liver regeneration. More importantly, our results show that PP2Aca-/- liver specific deletion induces dramatic up-regulated phosphorylation in glucagon signaling pathway, insulin signaling pathway, proteoglycans in cancer and AMPK signaling pathway, suggesting the critical role of PP2A in regulating termination of liver regeneration.

\title{
Conclusions
}

Based on our findings, we have revealed significant metabolic reprograming regulated by protein phosphorylation in the termination of liver regeneration. Further investigations on some crucial key metabolic enzymes or signal transduction molecules which are differentially phosphorylated should be carried out to elucidate the precise mechanisms regulating the termination of liver regeneration, which could greatly benefit the treatment of liver cancer via liver resection and donor liver transplantation.

\section{Supplementary Material}

Refer to Web version on PubMed Central for supplementary material.

\section{Acknowledgement}

\author{
We thank "Translational Medicine Core Facilities of Medical School of Nanjing University" for the use of mass \\ spectrometry facilities and bioinformatics analysis. \\ Funding Sources \\ This work was supported by the National Natural Science Foundation of China (31500664 and 31770838 to LF, \\ 31371373 and 31771572 to BX), the National Institute of Health (R01GM074830 to L.H.), the Natural Science \\ Foundation of Jiangsu Province (BK20171338 to LF and BK20151395 to BX), the Fundamental Research Funds \\ for the Central Universities (021414380334 to LF, 021414380330 to BX), and the Open Fund of State Key \\ Laboratory of Natural Medicines (SKLNMKF201811 to BX) and Six talent peaks project in Jiangsu Province \\ (yy-014 to BX).
}




\section{Abbreviations}

LR

PH

PTM

TMT

PP2Aca

HPLC

IMAC liver regeneration

partial hepatectomy

post-translational modification

Tandem Mass Tag

serine/threonine-protein phosphatase $2 \mathrm{~A}$ catalytic subunit alpha isoform

high performance liquid chromatography

immobilized metal ion affinity chromatography

\section{References}

1. Mao SA; Glorioso JM; Nyberg SL, Liver regeneration. Translational research : the journal of laboratory and clinical medicine 2014, 163, (4), 352-62. [PubMed: 24495569]

2. Cauchy F; Soubrane O; Belghiti J, Liver resection for HCC: patient's selection and controversial scenarios. Best practice \& research. Clinical gastroenterology 2014, 28, (5), 881-96. [PubMed: 25260315]

3. Bhandare MS; Patkar S; Shetty N; Polnaya A; Kulkarni S; Dusane RR; Shrikhande SV; Goel M, Liver resection for HCC outside the BCLC criteria. Langenbeck's archives of surgery 2018, 403, (1), 37-44.

4. Olle EW; Ren X; McClintock SD; Warner RL; Deogracias MP; Johnson KJ; Colletti LM, Matrix metalloproteinase-9 is an important factor in hepatic regeneration after partial hepatectomy in mice. Hepatology 2006, 44, (3), 540-9. [PubMed: 16941692]

5. Ishikawa T; Factor VM; Marquardt JU; Raggi C; Seo D; Kitade M; Conner EA; Thorgeirsson SS, Hepatocyte growth factor/c-met signaling is required for stem-cell-mediated liver regeneration in mice. Hepatology 2012, 55, (4), 1215-26. [PubMed: 22095660]

6. Liu G; Xie C; Fang Y; Qian K; Liu Q; Liu G; Cao Z; Du H; Fu J; Xu X, Splenectomy after partial hepatectomy accelerates liver regeneration in mice by promoting tight junction formation via polarity protein Par 3-aPKC. Life sciences 2018, 192, 91-98. [PubMed: 29166570]

7. Zhang F; Zhang J; Li X; Li B; Tao K; Yue S, Notch signaling pathway regulates cell cycle in proliferating hepatocytes involved in liver regeneration. Journal of gastroenterology and hepatology 2018, 8;33(8):1538-1547. [PubMed: 29384233]

8. Lu XF; Cao XY; Zhu YJ; Wu ZR; Zhuang X; Shao MY; Xu Q; Zhou YJ; Ji HJ; Lu QR; Shi YJ; Zeng $\mathrm{Y}$; Bu H, Histone deacetylase 3 promotes liver regeneration and liver cancer cells proliferation through signal transducer and activator of transcription 3 signaling pathway. Cell death \& disease 2018, 9, (3), 398. [PubMed: 29540666]

9. Huttlin EL; Jedrychowski MP; Elias JE; Goswami T; Rad R; Beausoleil SA; Villen J; Haas W; Sowa ME; Gygi SP, A tissue-specific atlas of mouse protein phosphorylation and expression. Cell 2010, 143, (7), 1174-89. [PubMed: 21183079]

10. Olsen JV; Blagoev B; Gnad F; Macek B; Kumar C; Mortensen P; Mann M, Global, in vivo, and site-specific phosphorylation dynamics in signaling networks. Cell 2006, 127, (3), 635-48. [PubMed: 17081983]

11. Ubersax JA; Ferrell JE Jr., Mechanisms of specificity in protein phosphorylation. Nature reviews. Molecular cell biology 2007, 8, (7), 530-41. [PubMed: 17585314]

12. Song C; Ye M; Liu Z; Cheng H; Jiang X; Han G; Songyang Z; Tan Y; Wang H; Ren J; Xue Y; Zou $\mathrm{H}$, Systematic analysis of protein phosphorylation networks from phosphoproteomic data. Molecular \& cellular proteomics : MCP 2012, 11, (10), 1070-83. [PubMed: 22798277] 
13. Grimsrud PA; Carson JJ; Hebert AS; Hubler SL; Niemi NM; Bailey DJ; Jochem A; Stapleton DS; Keller MP; Westphall MS; Yandell BS; Attie AD; Coon JJ; Pagliarini DJ, A quantitative map of the liver mitochondrial phosphoproteome reveals posttranslational control of ketogenesis. Cell metabolism 2012, 16, (5), 672-83. [PubMed: 23140645]

14. Wagih O; Sugiyama N; Ishihama Y; Beltrao P, Uncovering Phosphorylation-Based Specificities through Functional Interaction Networks. Molecular \& cellular proteomics : MCP 2016, 15, (1), 236-45. [PubMed: 26572964]

15. Humphrey SJ; James DE; Mann M, Protein Phosphorylation: A Major Switch Mechanism for Metabolic Regulation. Trends in endocrinology and metabolism: TEM 2015, 26, (12), 676-687.

16. Padrao AI; Vitorino R; Duarte JA; Ferreira R; Amado F, Unraveling the phosphoproteome dynamics in mammal mitochondria from a network perspective. Journal of proteome research 2013, 12, (10), 4257-67. [PubMed: 23964737]

17. Moroishi T; Park HW; Qin B; Chen Q; Meng Z; Plouffe SW; Taniguchi K; Yu FX; Karin M; Pan D; Guan KL, A YAP/TAZ-induced feedback mechanism regulates Hippo pathway homeostasis. Genes \& development 2015, 29, (12), 1271-84. [PubMed: 26109050]

18. Pauta M; Rotllan N; Fernandez-Hernando A; Langhi C; Ribera J; Lu M; Boix L; Bruix J; Jimenez W; Suarez Y; Ford DA; Baldan A; Birnbaum MJ; Morales-Ruiz M; Fernandez-Hernando C, Aktmediated foxo1 inhibition is required for liver regeneration. Hepatology 2016, 63, (5), 1660-74. [PubMed: 26473496]

19. Lu L; Finegold MJ; Johnson RL, Hippo pathway coactivators Yap and Taz are required to coordinate mammalian liver regeneration. Experimental \& molecular medicine 2018, 50, (1), e423. [PubMed: 29303509]

20. Bhushan B; Poudel S; Manley MW Jr.; Roy N; Apte U, Inhibition of Glycogen Synthase Kinase 3 Accelerated Liver Regeneration after Acetaminophen-Induced Hepatotoxicity in Mice. The American journal of pathology 2017, 187, (3), 543-552. [PubMed: 28068511]

21. Merlen G; Gentric G; Celton-Morizur S; Foretz M; Guidotti JE; Fauveau V; Leclerc J; Viollet B; Desdouets C, AMPKalpha1 controls hepatocyte proliferation independently of energy balance by regulating Cyclin A2 expression. Journal of hepatology 2014, 60, (1), 152-9. [PubMed: 24012615]

22. Kremer M; Son G; Zhang K; Moore SM; Norris A; Manzini G; Wheeler MD; Hines IN, Smad3 signaling in the regenerating liver: implications for the regulation of IL-6 expression. Transplant international : official journal of the European Society for Organ Transplantation 2014, 27, (7), 748-58. [PubMed: 24649805]

23. Karkampouna S; Goumans MJ; Ten Dijke P; Dooley S; Kruithof-de Julio M, Inhibition of TGFbeta type I receptor activity facilitates liver regeneration upon acute $\mathrm{CCl} 4$ intoxication in mice. Archives of toxicology 2016, 90, (2), 347-57. [PubMed: 25566828]

24. Kolupaeva V; Janssens V, PP1 and PP2A phosphatases--cooperating partners in modulating retinoblastoma protein activation. The FEBS journal 2013, 280, (2), 627-43. [PubMed: 22299668]

25. Lai SS; Zhao DD; Cao P; Lu K; Luo OY; Chen WB; Liu J; Jiang EZ; Yu ZH; Lee G; Li J; Yu DC; $\mathrm{Xu}$ XJ; Zhu MS; Gao X; Li CJ; Xue B, PP2Acalpha positively regulates the termination of liver regeneration in mice through the AKT/GSK3beta/Cyclin D1 pathway. Journal of hepatology 2016, 64, (2), 352-360. [PubMed: 26456844]

26. Zhou H; Ye M; Dong J; Corradini E; Cristobal A; Heck AJ; Zou H; Mohammed S, Robust phosphoproteome enrichment using monodisperse microsphere-based immobilized titanium (IV) ion affinity chromatography. Nature protocols 2013, 8, (3), 461-80. [PubMed: 23391890]

27. Vizcaino JA; Csordas A; Del-Toro N; Dianes JA; Griss J; Lavidas I; Mayer G; Perez-Riverol Y; Reisinger F; Ternent T; Xu QW; Wang R; Hermjakob H, 2016 update of the PRIDE database and its related tools. Nucleic acids research 2016, 44, (22), 11033. [PubMed: 27683222]

28. Chou MF; Schwartz D, Biological sequence motif discovery using motif-x. Current protocols in bioinformatics 2011, Chapter 13, Unit 13 15-24. [PubMed: 21901740]

29. Shen Z; Wang B; Luo J; Jiang K; Zhang H; Mustonen H; Puolakkainen P; Zhu J; Ye Y; Wang S, Global-scale profiling of differential expressed lysine acetylated proteins in colorectal cancer tumors and paired liver metastases. Journal of proteomics 2016, 142, 24-32. [PubMed: 27178108]

J Proteome Res. Author manuscript; available in PMC 2020 May 08. 
30. Zhang H; Hou J; Cui R; Guo X; Shi Z; Yang F; Dai J, Phosphoproteome analysis reveals an important role for glycogen synthase kinase-3 in perfluorododecanoic acid-induced rat liver toxicity. Toxicology letters 2013, 218, (1), 61-9. [PubMed: 23353032]

31. Forrest AR; Taylor DF; Fink JL; Gongora MM; Flegg C; Teasdale RD; Suzuki H; Kanamori M; Kai C; Hayashizaki Y; Grimmond SM, PhosphoregDB: the tissue and sub-cellular distribution of mammalian protein kinases and phosphatases. BMC bioinformatics 2006, 7, 82. [PubMed: 16504016]

32. Humphrey SJ; Azimifar SB; Mann M, High-throughput phosphoproteomics reveals in vivo insulin signaling dynamics. Nature biotechnology 2015, 33, (9), 990-5.

33. Akhmedov D; De Marchi U; Wollheim CB; Wiederkehr A, Pyruvate dehydrogenase E1alpha phosphorylation is induced by glucose but does not control metabolism-secretion coupling in INS-1E clonal beta-cells. Biochimica et biophysica acta 2012, 1823, (10), 1815-24. [PubMed: 22809973]

34. Fan J; Kang HB; Shan C; Elf S; Lin R; Xie J; Gu TL; Aguiar M; Lonning S; Chung TW; Arellano M; Khoury HJ; Shin DM; Khuri FR; Boggon TJ; Kang S; Chen J, Tyr-301 phosphorylation inhibits pyruvate dehydrogenase by blocking substrate binding and promotes the Warburg effect. The Journal of biological chemistry 2014, 289, (38), 26533-41. [PubMed: 25104357]

35. Colbert CL; Kim CW; Moon YA; Henry L; Palnitkar M; McKean WB; Fitzgerald K; Deisenhofer J; Horton JD; Kwon HJ, Crystal structure of Spot 14, a modulator of fatty acid synthesis. Proceedings of the National Academy of Sciences of the United States of America 2010, 107, (44), 18820-5. [PubMed: 20952656]

36. Heinrich G; Ghadieh HE; Ghanem SS; Muturi HT; Rezaei K; Al-Share QY; Bowman TA; Zhang D; Garofalo RS; Yin L; Najjar SM, Loss of Hepatic CEACAM1: A Unifying Mechanism Linking Insulin Resistance to Obesity and Non-Alcoholic Fatty Liver Disease. Frontiers in endocrinology 2017, 8, 8. [PubMed: 28184213] 

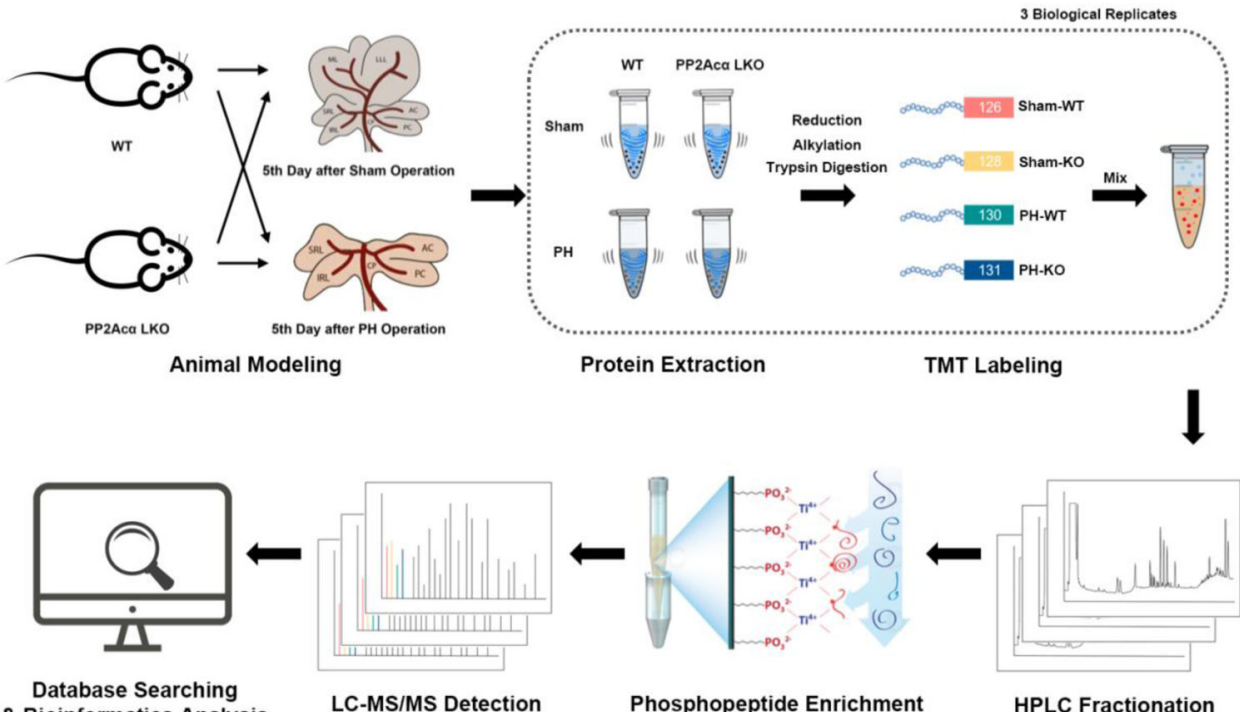
\& Bioinformatics Analysis

Figure 1.

A schematic workflow of phosphoproteomic analysis in the termination of liver regeneration in mice. 
A

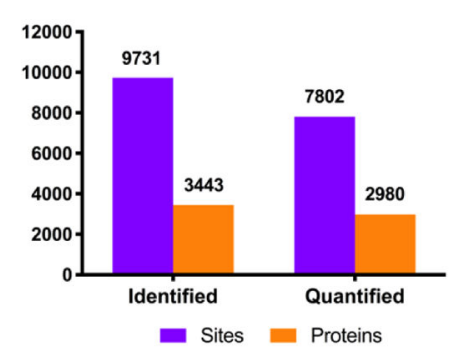

\begin{tabular}{cccc}
\hline Compared Groups & Type & $\begin{array}{c}\text { Up-regulated } \\
(>\mathbf{1 . 2})\end{array}$ & $\begin{array}{c}\text { Down-regulated } \\
(<\mathbf{0 . 8 8 3})\end{array}$ \\
\hline \multirow{2}{*}{ PH-KO/PH-WT } & Sites & 539 & 420 \\
& Proteins & 388 & 327 \\
\hline \multirow{2}{*}{ PH-KO/Sham-KO } & Sites & 483 & 320 \\
& Proteins & 364 & 230 \\
\hline \multirow{2}{*}{ PH-WT/Sham-WT } & Sites & 754 & 600 \\
& Proteins & 506 & 417 \\
\hline \multirow{2}{*}{ Sham-KO/Sham-WT } & Sites & 577 & 477 \\
& Proteins & 398 & 346 \\
\hline
\end{tabular}

Figure 2.

Quantification overview of phosphoproteomic analysis in the termination of liver regeneration in mice. A. Summary of identified and quantified phosphoproteins and phosphopeptides; B. Summary of drastically changed global phosphorylation in the termination of liver regeneration. 
A

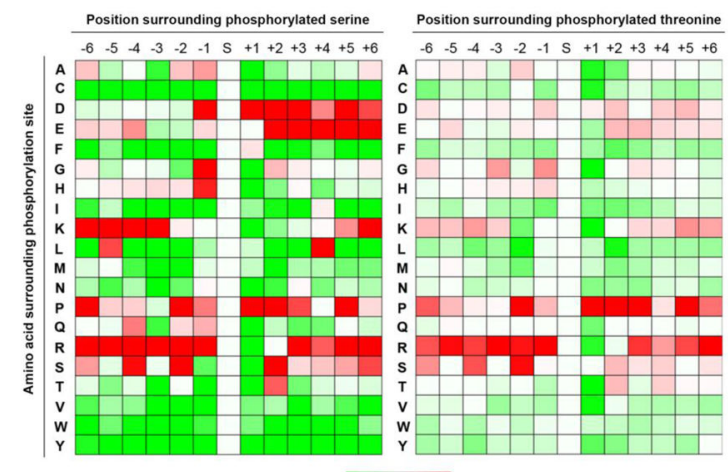

If fold change $<1 \quad$ if fold change $>1$

$\log 10$ (Fisher's exact test $P$ value) $-10 \begin{array}{llllll}-5 & 0 & 5 & 10 & -\log 10 \text { (Fisher's exact test } P \text { value) }\end{array}$

B

\begin{tabular}{|c|c|c|c|c|c|c|c|c|}
\hline \multirow{2}{*}{ Motit Logo } & \multirow{2}{*}{ Motif } & \multirow{2}{*}{$\begin{array}{l}\text { Moit } \\
\text { score }\end{array}$} & \multicolumn{2}{|c|}{ Foreground } & \multicolumn{2}{|c|}{ Background } & \multirow{2}{*}{$\begin{array}{c}\begin{array}{c}\text { Fold } \\
\text { Increase }\end{array} \\
\text {. }\end{array}$} & \multirow{2}{*}{ Putative Kinase } \\
\hline & & & Matches & size & Matches & size & & \\
\hline ce & ..RP.SP & 42.37 & 66 & 8270 & 343 & 760024 & 17.7 & ERKMAPK, CaMK-11 \\
\hline 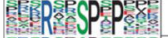 & ..R.SP.P. & 44.77 & 97 & 8204 & 352 & 750081 & 25.5 & Cank-il \\
\hline SPNice & R.SP & 32 & 249 & $\pi 52$ & 2359 & 780001 & 10.3 & cank-\|t \\
\hline rasp & ....PSPK... & 39.99 & 37 & 7503 & 207 & 755642 & 18 & ERKAMPK, COK-1 \\
\hline 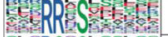 & RRS & 32 & 395 & 7053 & 3419 & 786590 & 12 & PKA, PKC, CaMk-II \\
\hline 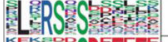 & LRS.S. & 46.91 & 87 & 6354 & 525 & 740005 & 19.3 & CaMK-II, CHK-I, NEK-VI, PDK \\
\hline & SO.E.E. & 38.29 & 59 & 5034 & 483 & 719368 & 17.5 & ck-11 \\
\hline - & ...DSEE... & 4.53 & 44 & 4911 & 390 & 717802 & 162 & cK-॥ \\
\hline 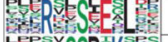 & R.S.EL & 39.08 & 20 & 4867 & 189 & 7174004 & 156 & Cank-II \\
\hline 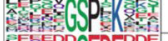 & $\ldots$....GSP.K... & 32.85 & 21 & 4847 & 95 & 717215 & 327 & COK-1 \\
\hline 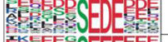 & SEDE. & 40.09 & 35 & 3006 & 412 & 670763 & 167 & cא-॥l \\
\hline 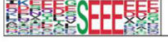 & S. SEEE. & 39.91 & 41 & 3371 & 709 & 670351 & 11.5 & cK-11 \\
\hline
\end{tabular}

c

\begin{tabular}{|c|c|c|c|c|c|c|c|c|}
\hline \multirow{2}{*}{ Motif Logo } & \multirow{2}{*}{ Moit } & \multirow{2}{*}{$\begin{array}{l}\text { Mout } \\
\text { score }\end{array}$} & \multicolumn{2}{|c|}{ Foreground } & \multicolumn{2}{|c|}{ Backround } & \multirow{2}{*}{$\begin{array}{c}\begin{array}{c}\text { Fold } \\
\text { Incroase }\end{array} \\
\text {. }\end{array}$} & \multirow{2}{*}{ Putative Kinase } \\
\hline & & & Matches & Sizo & Matches & Sizo & & \\
\hline 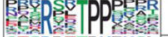 & R.TPP.... & 39.34 & 28 & 1136 & 156 & 482334 & 762 & Calle-ll \\
\hline Sto & RP.TP & 39.36 & 23 & 1108 & 180 & 482178 & 55.6 & CaMK-II, ERKMAPK \\
\hline 81 & .....TP.... & 32 & 120 & 1085 & 2482 & 481998 & 21.5 & ERKMAPK, COK-II \\
\hline 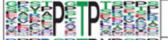 & P.PP. & 20.35 & 89 & 965 & 3041 & 479516 & 14.5 & ERKMAPK \\
\hline 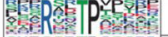 & R.TP..... & 28.46 & 54 & 876 & 1413 & 478475 & 20.8 & Conk-1I \\
\hline 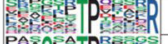 & TPR & 26.52 & ${ }_{45}$ & 822 & 1332 & 475062 & 19.5 & CDK-11 \\
\hline 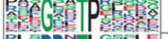 & .G.TP... & 22.93 & 41 & $m$ & 1723 & 473730 & 14.5 & COK-1 \\
\hline 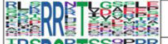 & RRT. & 28.78 & 33 & 545 & 1569 & 450005 & 17.4 & PKA, PKC, CaMK-II \\
\hline 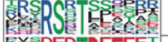 & .RS.T. . . & 25.95 & 27 & 512 & 1872 & 448436 & 126 & cank-il \\
\hline 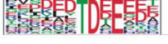 & Tr.e. & 18.83 & 21 & 453 & 1832 & 443661 & 112 & ck-11 \\
\hline
\end{tabular}

Figure 3.

Motif analysis of all identified phosphosites in the termination of liver regeneration in mice. A. The sequence motifs of 6 amino acids upstream and downstream of surrounding the phosphorylated serine or threonine sites; B. 12 Ser containing motifs with fold increase $>10$ and their putative kinases; C. $10 \mathrm{Thr}$ containing motifs with fold increase $>10$ and their putative kinases. 


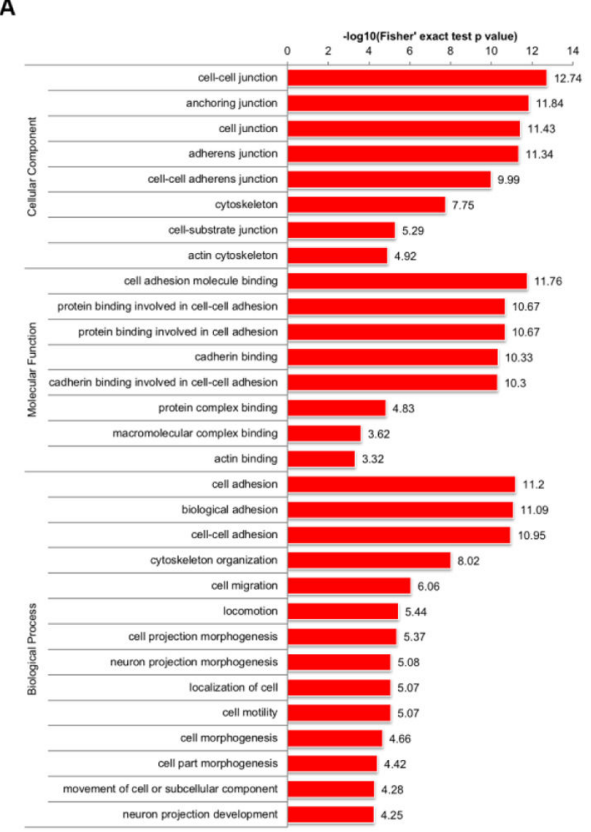

C

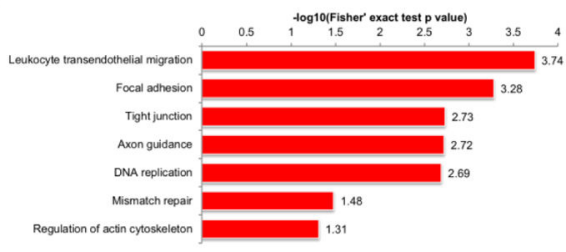

E

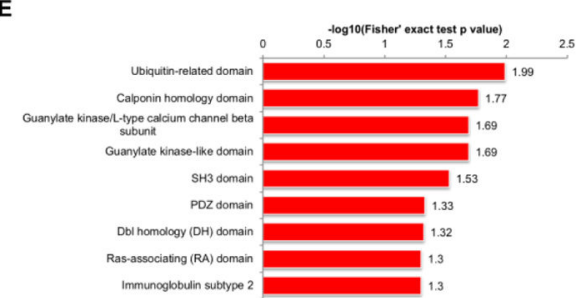

B

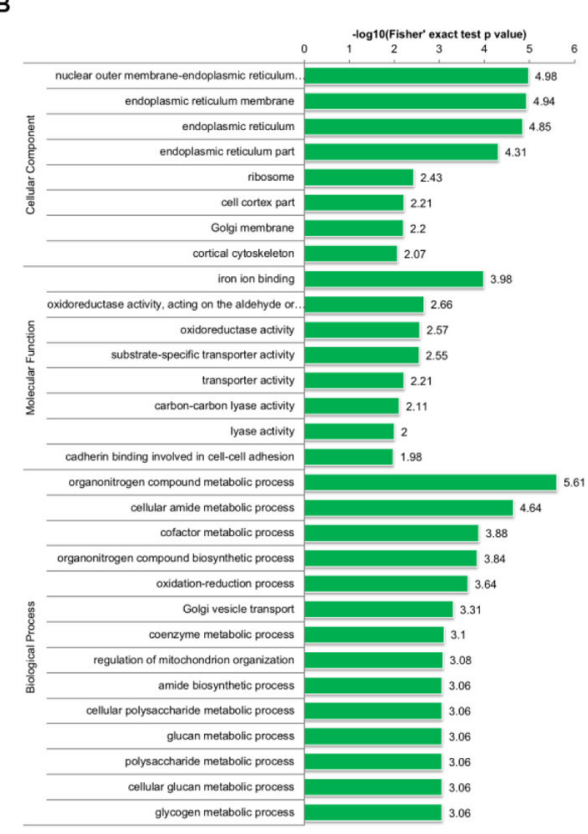

D

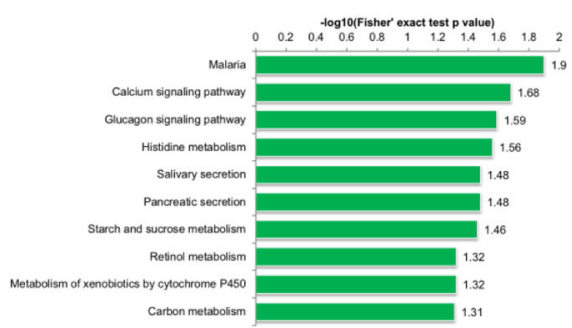

$\mathbf{F}$

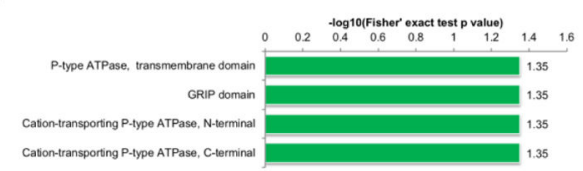

Figure 4.

Bioinformatics analysis of proteins with differentially quantified phosphosites in PH-WT/ Sham-WT. GO-based enrichment analysis of proteins with up-regulated phosphosites (A) and proteins with down-regulated phosphosites (B) in PH-WT/Sham-WT; KEGG pathwaybased enrichment analysis of proteins with up-regulated phosphosites (C) and proteins with down-regulated phosphosites (D) in PH-WT/Sham-WT; Protein domain enrichment analysis of proteins with up-regulated phosphosites (E) and proteins with down-regulated phosphosites (F) in PH-WT/Sham-WT. 
A

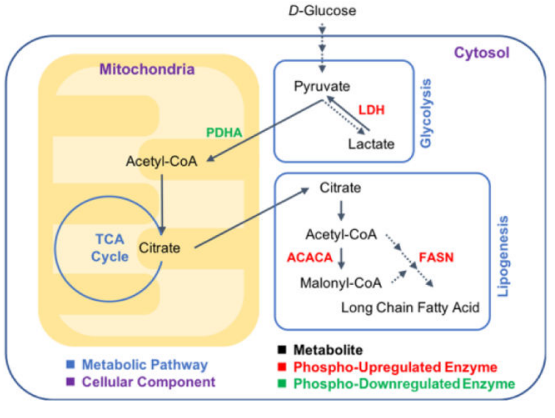
IP: 6BE1

$F$

G c

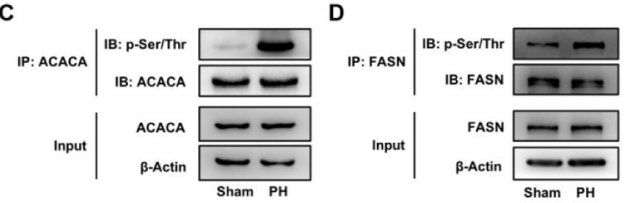

G

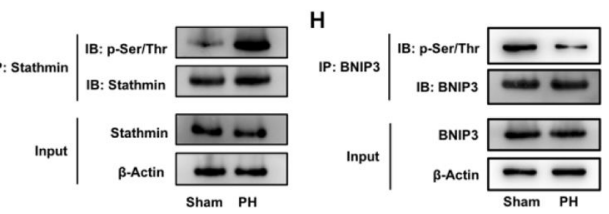

Figure 5.

Validation of proteins with differentially quantified phosphosites in PH-WT/Sham-WT. A. The significantly changed metabolic pathways obtained from KEGG pathway enrichment analysis in PH-WT/Sham-WT. B-H: Validation of significantly changed phosphorylation of PDHA, ACACA, FASN, GBE1, MAP4K4, Stathmin and BNIP3 in PH-WT/Sham-WT, respectively. 


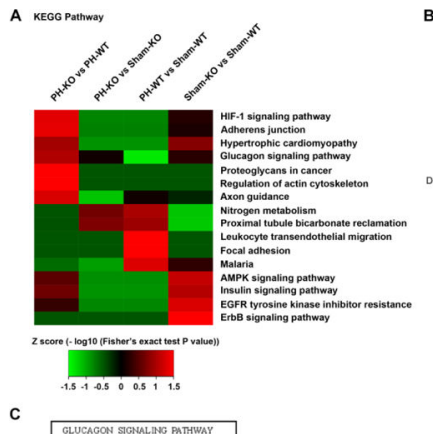

B
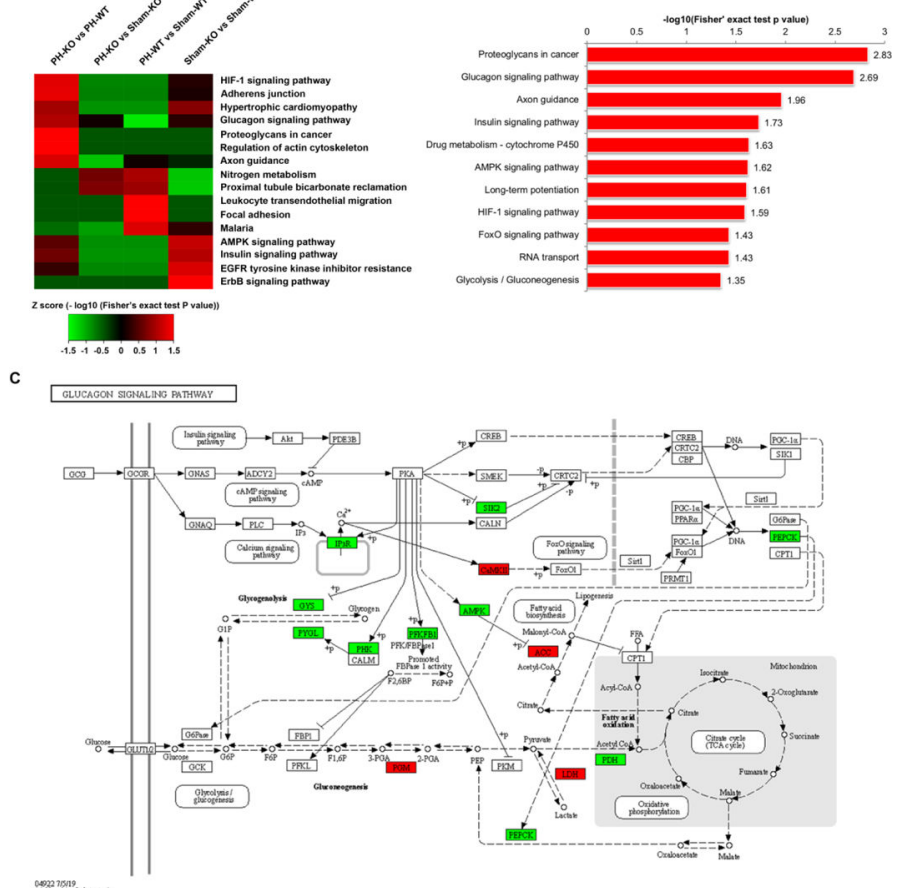

,
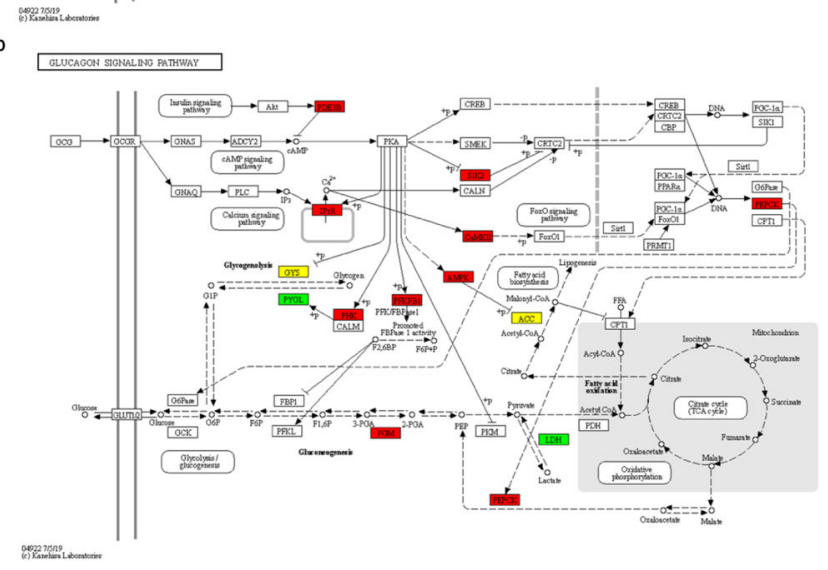

Figure 6.

PP2Aca is essential for the proper termination of liver regeneration after partial hepatectomy through dephosphorylation of key molecules in multiple signaling pathways. A. Dramatic global phosphorylation changes in PP2Aca-/- mice compared to wild type mice in the termination of liver regeneration, as shown by enrichment-based clustering on KEGG pathways. B. KEGG pathways with markedly up-regulated phosphorylation in $\mathrm{PH}-$ $\mathrm{KO} / \mathrm{PH}-\mathrm{WT}$ in the termination of liver regeneration. C-D. In contrary to the efficient downregulation in wild type mice to facilitate the termination of liver regeneration $(\mathrm{C})$, glucagon signaling pathway is consistently activated in PP2Aca-/- mice at day 5 after PH (D). 